\title{
Sleep Dysfunction in Patients with Cancer
}

\author{
Lavinia Fiorentino, MS and Sonia Ancoli-Israel, PhD
}

\section{Opinion statement}

Sleep complaints are common in cancer patients. Insomnia is particularly a concern in this population. Although pharmacotherapy is the most prescribed treatment for sleep disturbances, there is evidence that cognitive-behavioral therapy (CBT) is an effective treatment for insomnia in all patients, including those with cancer. CBT for insomnia is a flexible treatment, tailored to the needs of a specific patient, and focusing on behavioral and psychologic skills that foster better sleep and lower anxiety. Many cancer patients with insomnia may be hesitant to use drugs for their sleep treatment because they are already overwhelmed by the chemical and pharmacologic treatments they are prescribed for the cancer; thus, CBT may become the treatment of choice for insomnia in these patients.

\section{Introduction}

The sleep of cancer patients is often disturbed [1], yet sleep disorders and their health consequences are often neglected in this population [2]. Most of the literature looking at sleep in cancer patients has focused on insomnia, particularly in breast cancer patients [3], with a few studies exploring the prevalence of sleep-disordered breathing (SDB) in head and neck cancer patients [4, Class III]. Systematic research on other sleep disorders is lacking in cancer patients. Understanding the relationship between sleep disorders and cancer is important because the physical and psychological correlates of poor sleep may interact with the patient's cancer treatment, general well-being, and quality of life.

\section{PREVALENCE OF SLEEP DISTURBANCES IN CANCER}

Sleep disturbances affect between $30 \%$ to $75 \%$ of newly diagnosed or recently treated cancer patients [1], a rate that has been reported as double that of the general population [5••]. Surveys have suggested that sleep complaints in cancer patients consisted of difficulty falling asleep and difficulty staying asleep, with frequent and prolonged nighttime awakenings. Patients reported these complaints both before treatment [6] and during treatment [7, Class III]. A study comparing cancer patients with psychiatric patients and controls found that $62 \%$ of the cancer patients reported moderate to severe sleep disturbance, whereas 53\% of depressed patients and only $30 \%$ of the volunteers reported sleep disturbance [8, Class II]. There are very few studies investigating sleep disturbances among advanced cancer patients. One study reported that $72 \%$ of patients with advanced cancers reported sleep disturbances [9, Class III]. The most common complaints were difficulty falling asleep (40\%), difficulty staying asleep (63\%), and not feeling rested in the morning (72\%). Furthermore, $19 \%$ of the cancer patients reported having trouble sleeping before their cancer diagnosis.

Sleep disturbance in cancer patients might be underestimated. A telephone survey by Engstrom et al. [7, Class III] in 150 lung or breast cancer patients in various stages of treatment and who

Corresponding author: Sonia Ancoli-Israel, PhD, Department of Psychiatry 0603, University of California San Diego, 9500 Gilman Drive, La Jolla, CA 92093, USA. sancoliisrael@ ucsd.edu. 
were undergoing a variety of treatments revealed that $44 \%$ reported a sleep problem in the previous month, but only approximately $17 \%$ communicated the problem to their doctors. This study also surveyed a second group of cancer patients and found that $45 \%$ reported a sleep problem in the prior month, and of these $50 \%$ rated the sleep problem as moderate, severe, or intolerable. The sleep complaints reported were varied, with $90 \%$ of the patients complaining of awakening during the night, approximately $85 \%$ complaining of sleeping fewer hours than normal, $75 \%$ complaining of difficulty in getting back to sleep, and 39\% reporting napping at unusual times, such as midmorning and mid-afternoon.

A survey by Davidson et al. [10, Class III] conducted in 1000 cancer patients with different types of cancer and at different treatment phases found that $31 \%$ reported insomnia, $28 \%$ reported excessive sleepiness, and $41 \%$ complained of restless legs. In this study, the prevalence of sleep problems varied among different types of cancer: lung cancer patients had the highest or second-highest prevalence of sleep problems in general, whereas breast cancer patients had a high prevalence of insomnia and fatigue. Another survey found that the amount of insomnia in cancer patients was as high as the amount of insomnia in depressed patients [11, Class III]. Savard et al. [12, Class III] looked at the prevalence of insomnia in 300 women with breast cancer and found that 19\% met the diagnostic criteria for insomnia, with $95 \%$ of the cases being chronic insomnia. In addition, in 33\% of the cases the onset of insomnia followed the breast cancer diagnosis, and 58\% of the patients reported that cancer either caused or aggravated their sleep problems.

\section{OBJECTIVE SLEEP MEASURES}

The gold standard for recording sleep is polysomnography (PSG), consisting of overnight sleep measurements of brain waves, eye movement, muscle tension, and often respiration, heart rate, and leg movements. Although not invasive, PSG recordings can be cumbersome, particularly for cancer patients who are already fatigued or in pain. Few studies have used PSG to study sleep in cancer.

Silberfarb et al. [13, Class II] compared the PSG recordings of lung cancer patients, breast cancer patients, insomnia patients, and normal volunteers. They found that the patients with insomnia had the shortest total sleep time of all the groups and that the lung cancer patients had the most disrupted sleep, characterized by spending more time in bed without sleeping, lower sleep efficiency (the percentage of time in bed actually spent asleep), longer sleep-onset latency (time to fall asleep), and more time awake during the night, compared with the other groups.

The same study found that none of the cancer patients had SDB but that there was a higher prevalence of periodic limb movements in sleep (PLMS) in the cancer patients, compared with controls or insomnia patients. Data from our laboratory showed that $36 \%$ of breast cancer patients had PLMS [14, Class III]. Because PLMS is treatable, these data suggest that it would be important to rule out PLMS as a cause of sleep disturbance in patients with cancer.

Our laboratory also collected PSG recordings post-treatment in breast cancer patients and found disturbed sleep, with more time spent in lighter stages of sleep (stages 1 and 2) and less time spent in deep stages (stages 3 and 4) or rapid eye movement (REM) sleep. These women also spent more time awake, with lower sleep efficiency (amount of sleep given the amount of time in bed) compared with the norms of the general population [15, Class III]. Savard et al.

$[16 \bullet, 17$, Class III] performed PSG in breast cancer patients and found that hot flashes were associated with sleep disturbances in patients complaining of insomnia, with nights with hot flashes significantly worse than nights without hot flashes (increased wake time, lower percentage of stage 2 sleep, and longer REM latency). 
Because PSGs can be difficult to record, many researchers have used actigraphy to study sleep/ wake patterns. An actigraph is a device about the size of a wrist watch, worn on the wrist, that records movement by using motion-sensitive accelerometers [18]. Special algorithms estimate sleep and wake time from the movement, and correlation studies with PSG recordings show high reliability.

Miaskowski and Lee [19, Class III] recorded actigraphy over a 48-hour period in 24 patients at various times during radiation therapy for bone metastases. They found that as radiation therapy progressed, the complaints of poor sleep increased. Furthermore, sleep efficiency decreased, and frequent urination, rather than pain intensity, was reported to be the main cause of nighttime awakenings. In a pilot study using actigraphy, Payne et al. [20, Class II] reported that breast cancer patients had significantly shorter total sleep time, compared with healthy controls.

Our laboratory studied actigraphic sleep variables and patient reports of sleep quality in 82 women before and during chemotherapy for breast cancer. Results showed that patients complained of sleep problems before the start of chemotherapy [21, Class III], with baseline measurements suggesting the women were asleep on average for $77 \%$ of the night (mean $=6.1$ hours asleep and mean $=1.9$ hours awake). In the same study, disturbed sleep was correlated with fatigue, depressive symptoms, and functional outcome [21, Class III]. A causal relationship between sleep and these factors could not be determined from this study. However, the pretreatment sleep disturbance predicted more fatigue, more depressive symptoms, and worse quality of life throughout the chemotherapy [22, Class III]. These data suggest that sleep disturbances in cancer patients may be a result of multiple factors, including the cancer itself or cancer-related symptoms. However, psychologic factors have also been shown to predict sleep disturbance. One study showed that the sleep disturbances in metastatic breast cancer patients can be predicted by psychologic factors at baseline, such as depression, perception of pain, and life stressors, and that the worsening of sleep over time was strongly associated with the worsening of pain and depression [23, Class III].

\section{ETIOLOGY OF SLEEP DISTURBANCES IN CANCER}

There are multiple causes of sleep disruption in cancer patients. Based on Speilman's threefactor model of insomnia [24], which states that there are predisposing, precipitating, and perpetuating factors, Savard and Morin [2] hypothesized that in cancer 1) predisposing factors that increase the individual's general vulnerability to develop insomnia might be hyperarousability, female gender, aging, and a personal and a familial history of insomnia; 2) precipitating factors that trigger the onset of sleep disturbances might be the cancer itself, cancer-related emotional impact and functional loss, and cancer-related treatments, pain, and delirium; and 3) perpetuating factors that contribute to the maintenance of sleep disturbance over time might be maladaptive sleep behaviors and faulty beliefs and attitudes about sleep.

Most of the studies of sleep in cancer have been conducted in women with breast cancer. The population of women with breast cancer is possibly more prone to insomnia for various reasons, including disruption of sleep due to increased frequency and severity of the hot flashes associated with sudden menopause secondary to the breast cancer treatment, the fact that depression is more prevalent in women and there is a strong association between depression and insomnia, and the fact that insomnia is more common in women than in men. Savard et al. [12, Class III], in a study of insomnia, clinical characteristics, and risk factors in 300 breast cancer patients, found that factors associated with high risk of insomnia were sick leave, unemployment, widowhood, lumpectomy, chemotherapy, and a less severe stage of cancer at diagnosis. In patients with advanced cancer, lower performance status, anxiety, depression, and confusion were reported to be associated with sleep disturbance. Davidson et al. [10, Class 
III], in the large-scale survey mentioned earlier, found that insomnia-related risk factors included fatigue, age, restless legs, sedative/hypnotic use, low or variable mood, dreams, concerns, and recent cancer surgery.

Radiation and chemotherapy are both known to produce sleep disturbances. However, as mentioned earlier, studies have shown that often sleep disturbances are present before the start of treatment. For example, Cimprich [6] administered self-report items relating to sleep quality, fatigue, and distress to breast cancer patients who had not yet undergone treatment and found that insomnia was correlated with high levels of distress and was the most frequent symptom, with $88 \%$ of the sample reporting difficulty sleeping. In this study, subjective reports of distress and anxiety were correlated with insomnia, and self-ratings of fatigue and sleep difficulty were high. In patients whose self-ratings of anxiety (as well as anger) were low, levels of insomnia and fatigue were still high. This contrasts with the general notion that disturbed sleep before treatment is attributable to the increased anxiety and stress accompanying the recent cancer diagnosis.

Other possible causes of disturbed sleep in cancer patients are pain and psychiatric disorders (eg, depression and anxiety) [25]. Engstrom et al. [7] hypothesize that pain is the cause of nocturnal awakenings and that the psychologic distress contributes to the difficulty falling asleep. Lewin and Dahl [26] discuss the relationship between pain in a variety of medical conditions and sleep. They theorized that because sleep leads to recovery and repair of tissue and may offer a temporary cessation of the psychologic awareness of pain, poor sleep can lead to decreased capacity to manage pain. Consequently, the cycle of pain and poor sleep becomes self-perpetuating. However, there is little empirical evidence that supports the notion that pain leads to disrupted sleep. Silberfarb et al. [13, Class II] compared 32 cancer patients (15 breast cancer, 17 lung cancer) with 32 age- and sex-matched normal volunteers and 32 patients with insomnia and found that only breast cancer patients complained of pain before bedtime; however, their sleep quality was not significantly affected. In addition, the poor sleep quality of the insomnia and lung cancer patients was not associated with reports of pain.

Cancer patients are often prescribed opioids for their pain. A common side effect of opioids is sedation. However, the relationship between opioid use and sleep has not been well-studied. A recent study by Dimsdale et al. [27, Class I] suggests that opioids decrease REM and slowwave sleep. Hence, rather than improving sleep, opioids may in fact be contributing to the sleep disturbances in cancer patients with chronic pain. Furthermore, respiratory depression is a common and serious side effect of opioids and might exacerbate the apneic events in cancer patients with SDB.

The relationship between sleep disturbances, depressive symptoms, and cancer remains unexplained. As mentioned earlier, it is well-known that insomnia is often comorbid with depression, that sleep disturbance is a risk factor of depressive symptoms, and that the amount of insomnia in cancer patients is comparable to the amount of insomnia found in depressed patients. However, several studies revealed that depression and sleep disturbances already exist before the start of any cancer treatment [6;21, Class III]. Whether the disruptions in sleep are secondary to or comorbid with pain and/or the medications used to mitigate the pain, anxiety, and depression remains unclear. Additional well-controlled longitudinal research studies are needed to answer these questions.

\section{BIOLOGICAL RHYTHMS}

Little is known about the links between circadian rhythms and sleep in cancer [28]. Studies suggest that cancer itself may be a result of disturbed biological rhythms [29]. The disruptions in biological rhythmicity are relevant to cancer, to the mitotic properties (cell division) of cancerous cells, to the treatments of cancer, and to the timing of the administration of the 
treatment, in addition to the quality of life in cancer patients. Hence, understanding the biological rhythms of cancer patients is crucial, and actigraphy (described earlier) represents a convenient, nonintrusive, and effective instrument to measure circadian activity rhythms in cancer populations.

In a study using actigraphy, Mormont et al. [30, Class III] investigated the circadian rhythm of the rest/activity cycle and of serum cortisol, leukocyte counts, and neutrophil counts for 3 consecutive days before beginning chronomodulated chemotherapy in patients with metastatic colorectal cancer. They found that patients with marked activity rhythms (ie, greater activity when out of bed than when in bed) had a fivefold higher survival at 2-year follow-up than those with less synchronized rhythms. Patients with marked activity rhythms also had better quality of life and reported significantly less fatigue. Circadian rhythms in activity and in leukocytes combined were prognostic of response. The researchers concluded that the rest/activity cycle can be used to predict prognosis for cancer patients' survival and tumor response.

There is evidence of a strong relationship between sleep disturbances and interrupted circadian rhythms in cancer patients. Liu et al. [31] used actigraphy to measure circadian activity rhythms and sleep/wake patterns in breast cancer patients. The circadian rhythms were robust at baseline but desynchronized during the chemotherapy. The desynchronization, which was characterized by a disrupted rhythm, less activity both during the day and night, and a more delayed phase $(P=0.029-0.0066)$, was correlated with fatigue, low daytime light exposure, and decreased quality of life. Causality cannot be determined from this study; however, the data suggest that although fatigue may worsen secondary to chemotherapy, it may be mediated, at least in part, by the disrupted circadian rhythms. Future studies evaluating the effect of shifting circadian rhythms on fatigue in breast cancer patients are warranted.

In addition to sleep/wake activity, circadian rhythms may affect tissues and cell activity. Certain tumor types appear to undergo mitosis at specific times of day. In some cases, tumor cell mitosis appears to desynchronize from the patient's circadian rhythms. In other words, there are indications that the tumor as well as the host (ie, the patient) show signs of abnormal circadian rhythmicity as the cancer progresses. This rhythmicity may be used to develop new therapeutic methods (ie, chronotherapy). Chronotherapy involves choosing the timing of administration of a drug/treatment in order to minimize its side effects and maximize the therapeutic effects.

It has been documented that both toxicity and antitumor activity of cancer drugs are timedependent. Chronotherapy has been studied in cancer by Levi [32, Class I], who has shown that it is more effective and has lower toxicity and fewer side effects than regular treatment. Other reported benefits include enhanced quality of life and fewer days of hospitalization [33].

\section{INFLAMMATORY MARKERS}

Inflammation is a critical component of tumor progression. Cytokines are non-antibody polypeptides secreted by inflammatory leukocytes and some nonleukocytic cells and act as intercellular mediators. Cytokines are induced by infectious agents and can also be induced by cancer cells. Increased levels of cytokines have been found in the blood, ascites, pleural effusions, and urine of cancer patients. There is evidence that increased cyto-kines in cancer patients are correlated with circadian parameters [34, Class III]. Studies suggest that cancerrelated symptom clusters, such as sleep disturbance, circadian rhythms patterns, pain, fatigue, affective symptoms, and cognitive dysfunction, may share common cytokine-based neuroimmunologic mechanisms [35]. Hence, cytokines may also play a role in the sleep disturbances of cancer patients. 
Some cytokines appear to be directly correlated to sleep disturbances. Excessive daytime sleepiness, for example, is associated with increased plasma cytokines, and sleep deprivation can cause increase of proinflammatory cytokine levels. Inversely, a good night's sleep, or even a daytime nap after one night's sleep loss, can decrease the secretion of interleukin (IL)-6 $[36$, Class I].

Data from our laboratory showed that inflammatory markers such as vascular endothelial growth factor (VEGF) and soluble intercellular adhesion molecule-1 were increased during chemotherapy in breast cancer patients [37, Class III], and that the increased VEGF levels were associated with more disrupted sleep during chemotherapy [38, Class III].

Savard et al. [39••, Class I] looked at the relationship between insomnia and the immune system in breast cancer survivors. The analysis revealed that after successful treatment of the insomnia with cognitive-behavioral therapy (CBT), participants had higher cytokine production (ie, IL-1- $\beta$, interferon $\gamma$ ) and lower increases of lymphocytes, which then increased at follow-up, as did the leukocyte counts. These results corroborate the notion that sleep and immune regulation are related and that amelioration of insomnia symptoms can booster the immune system.

Notably, sleep disturbances can directly result from the cytokines' effect on the brain, or may be secondary to the cytokine-induced "sickness behavior" (eg, fatigue, fever, and disrupted circadian rhythms) [40]. Cytokines have been studied widely in cancer patients, but further research to understand the mechanisms of sleep disturbances and other cancer-related symptoms is needed. This research may lead to new treatments for these complaints.

\section{Acknowledgments}

Supported by NCI CA112035, CBCRP 11IB-0034, CBCRP 11GB-0049, NIH M01 RR00827, Moores UCSD Cancer Center, and the research service of the Veterans Affairs San Diego Health Care System.

\section{References and Recommended Reading}

Papers of particular interest, published recently, have been highlighted as:

- Of importance

•• Of major importance

1. Ancoli-Israel S, Moore P, Jones V. The relationship between fatigue and sleep in cancer patients: a review. Eur J Cancer Care (Engl) 2001;10:245-255. [PubMed: 11806675]

2. Savard J, Morin CM. Insomnia in the context of cancer: a review of a neglected problem. J Clin Oncol 2001;19:895-908. [PubMed: 11157043]

3. Fiorentino L, Ancoli-Israel S. Insomnia and its treatment in women with breast cancer. Sleep Med Rev 2006;10:419-429. [PubMed: 16963293]

4. Nesse W, Hoekema A, Stegenga B, et al. Prevalence of obstructive sleep apnoea following head and neck cancer treatment: a cross-sectional study. Oral Oncol 2006;42:108-114. [PubMed: 16150632]

5••. Berger AM, Parker KP, Young-McCaughan S, et al. Sleep wake disturbances in people with cancer and their caregivers: state of the science. Oncol Nurs Forum 2005;32:E98-E126. A comprehensive review. [PubMed: 16270104]

6. Cimprich B. Pretreatment symptom distress in women newly diagnosed with breast cancer. Cancer Nurs 1999;22:185-194. [PubMed: 10376379]

7. Engstrom CA, Strohl RA, Rose L, et al. Sleep alterations in cancer patients. Cancer Nurs 1999;22:143148. [PubMed: 10217030] 
8. Anderson KO, Getto CJ, Mendoza TR, et al. Fatigue and sleep disturbance in patients with cancer, patients with clinical depression, and community-dwelling adults. J Pain Symptom Manage 2003;25:307-318. [PubMed: 12691682]

9. Sela RA, Watanabe S, Nekolaichuk CL. Sleep disturbances in palliative cancer patients attending a pain and symptom control clinic. Palliat Support Care 2005;3:23-31. [PubMed: 16594191]

10. Davidson JR, MacLean AW, Brundage MD, Schulze K. Sleep disturbance in cancer patients. Soc Sci Med 2002;54:1309-1321. [PubMed: 12058848]

11. Holland JC, Plumb M. A comparative study of depressive symptoms in patients with advanced cancer. Proc Am Assoc Cancer Res 1977;18:201.

12. Savard J, Simard S, Blanchet J, et al. Prevalence, clinical characteristics, and risk factors for insomnia in the context of breast cancer. Sleep 2001;24:583-590. [PubMed: 11480655]

13. Silberfarb PM, Hauri PJ, Oxman TE, Schnurr PP. Assessment of sleep in patients with lung cancer and breast cancer. J Clin Oncol 1993;11:997-1004. [PubMed: 8487063]

14. Ancoli-Israel, S. Sleep and fatigue in cancer patients. In: Kryger, MH.; Roth, T.; Dement, WC., editors. Principles and Practice of Sleep Medicine. Philadelphia: WB Saunders; 2005. p. 1218-1224.

15. Fiorentino L, Mason W, Parker B, et al. Sleep disruption in breast cancer patients post-chemotherapy. Sleep 2005;28:A294.

16••. Savard J, Simard S, Ivers H, Morin CM. Randomized study on the efficacy of cognitive-behavioral therapy for insomnia secondary to breast cancer, part I: sleep and psychological effects. J Clin Oncol 2005;23:6083-6096. A well-conducted study. [PubMed: 16135475]

17. Savard J, Davidson JR, Ivers H, et al. The association between nocturnal hot flashes and sleep in breast cancer survivors. J Pain Symptom Manage 2004;27:513-522. [PubMed: 15165649]

18. Ancoli-Israel S, Cole R, Alessi CA, et al. The role of actigraphy in the study of sleep and circadian rhythms. Sleep 2003;26:342-392. [PubMed: 12749557]

19. Miaskowski C, Lee KA. Pain, fatigue and sleep disturbances in oncology outpatients receiving radiation therapy for bone metastasis: a pilot study. J Pain Symptom Manage 1999;17:320-332. [PubMed: 10355211]

20. Payne JK, Piper B, Rabinowitz I, Zimmerman B. Biomarkers, fatigue, sleep, and depressive symptoms in women with breast cancer: a pilot study. Oncol Nurs Forum 2006;33:775-783. [PubMed: 16858459]

21. Ancoli-Israel S, Liu L, Marler M, et al. Fatigue, sleep and circadian rhythms prior to chemotherapy for breast cancer. Support Care Cancer 2006;14:201-209. [PubMed: 16010529]

22. Liu L, Parker B, Dimsdale J, et al. Pre-treatment subjective sleep quality predicts fatigue, mood, and quality of life in breast cancer patients during chemotherapy. Sleep 2006;29(Suppl):A309.

23. Palesh OG, Collie K, Batiuchok D, et al. A longitudinal study of depression, pain, and stress as predictors of sleep disturbance among women with metastatic breast cancer. Biol Psychol 2007;75:37-44. [PubMed: 17166646]

24. Speilman, AJ.; Glovinsky, PB. Case studies in insomnia. In: Hauri, PJ., editor. The Varied Nature of Insomnia. New York: Plenum Press; 1991. p. 1-15.

25. Hu D, Silberfarb PM. Management of sleep problems in cancer patients. Oncology 1991;5:23-27. [PubMed: 1835874]

26. Lewin DS, Dahl RE. Importance of sleep in the management of pediatric pain. J Dev Behav Pediatr 1999;20:244-252. [PubMed: 10475599]

27. Dimsdale JE, Norman D, DeJardin D, Wallace MS. The effect of opioids on sleep architecture. J Clin Sleep Med 2007;3:33-36. [PubMed: 17557450]

28. Lee K, Cho M, Miaskowski C, Dodd M. Impaired sleep and rhythms in persons with cancer. Sleep Med Rev 2004;8:199-212. [PubMed: 15144962]

29. Moser M, Schaumberger K, Schernhammer E, Stevens RG. Cancer and rhythm. Cancer Causes Control 2006;17:483-487. [PubMed: 16596301]

30. Mormont MC, Waterhouse J, Bleuzen P, et al. Marked 24-h rest/activity rhythms are associated with better quality of life, better response and longer survival in patients with metastatic colorectal cancer and good performance status. Clin Cancer Res 2000;6:3038-3045. [PubMed: 10955782] 
31. Liu L, Marler M, Parker BA, et al. The relationship between fatigue and light exposure during chemotherapy. Support Care Cancer 2005;13:1010-1017. [PubMed: 15864659]

32. Levi F. Chronotherapeutics: the relevance of timing in cancer therapy. Cancer Causes Control 2006;17:611-621. [PubMed: 16596317]

33. Wood PA, Hrushesky WJM. Circadian rhythms and cancer chemotherapy. Crit Rev Eukaryot Gene Expr 1996;6:299-343. [PubMed: 8959371]

34. Rich T, Innominato PF, Boerner J, et al. Elevated serum cytokines correlated with altered behavior, serum cortisol rhythm, and dampened 24-hour rest-activity patterns in patients with metastatic colorectal cancer. Clin Cancer Res 2005;11:1757-1764. [PubMed: 15755997]

35. Lee BN, Dantzer R, Langley KE, et al. A cytokine-based neuroimmunologic mechanism of cancerrelated symptoms. Neuroimmunomodulation 2004;11:279-292. [PubMed: 15316238]

36. Vgontzas AN, Pejovic S, Zoumakis E, et al. Daytime napping after a night of sleep loss decreases sleepiness, improves performance, and causes beneficial changes in cortisol and interleukin-6 secretion. Am J Physiol Endocrinol Metab 2006;292:E253-E261. [PubMed: 16940468]

37. Mills PJ, Parker BA, Dimsdale JE, et al. The relationship between fatigue, quality of life and inflammation during anthracycline-based chemotherapy in breast cancer. Biol Psychol 2005;69:8596. [PubMed: 15740827]

38. Mills PJ, Parker BA, Jones V, et al. The effects of standard anthracycline-based chemotherapy on soluble ICAM-1 and vascular endothelial growth factor levels in breast cancer. Clin Cancer Res 2004;10:4998-5003. [PubMed: 15297400]

39••. Savard J, Simard S, Ivers H, Morin CM. Randomized study on the efficacy of cognitive-behavioral therapy for insomnia secondary to breast cancer, part II: immunologic effects. Clin Oncol 2005;23:6097-6106. A well-conducted study.

40. Kelley KW, Bluthe RM, Dantzer R, et al. Cytokine-induced sickness behavior. Brain Behav Immun 2003;17:S112-S118. [PubMed: 12615196]

41. Derogatis LR, Feldstein M, Morrow G, et al. A survey of psychotropic drug prescriptions in an oncology population. Cancer 1979;44:1919-1929. [PubMed: 40688]

42. Stiefel FC, Kornblith AB, Holland JC. Changes in the prescription patterns of psychotropic drugs over a 10-year period. Cancer 1990;65:1048-1053. [PubMed: 1967546]

43. National Institutes of Health State of the Science Conference Statement on Manifestations and Management of Chronic Insomnia in Adults, June 13-15, 2005. Sleep 2005;28:1049-1058. [PubMed: 16268373]

44. Bastien CH, Morin CM, Ouellet MC, et al. Cognitive-behavioral therapy for insomnia: comparison of individual therapy, group therapy, and telephone consultations. J Consult Clin Psychol 2004;72:653-659. [PubMed: 15301650]

45. Quesnel C, Savard J, Simard S, et al. Efficacy of cognitive-behavioral therapy for insomnia in women treated for nonmetastatic breast cancer. J Consult Clin Psychol 2003;71:189-200. [PubMed: 12602439]

46. Morin CM, Colecchi C, Stone J, et al. Behavioral and pharmacological therapies for late life insomnia. JAMA 1999;281:991-999. [PubMed: 10086433]

47. Theobald DE. Cancer pain, fatigue, distress, and insomnia in cancer patients. Clin Cornerstone 2004;6:S15-S21. [PubMed: 15675653]

\section{Treatment \\ Pharmacotherapy}

- Sleep disturbances in the general population are most commonly treated with pharmacotherapy. According to Derogatis et al. [41, Class III], hypnotics are the most common medications prescribed to cancer patients, accounting for $48 \%$ of the total prescriptions, with "sleep" as the physician's stated reason for the prescription in $85 \%$ of the 814 total prescriptions analyzed. Similar results were found in another study, with $44 \%$ of approximately 400 prescriptions over 200 consecutive cancer clinic patients written for hypnotic medications [42, Class III]. This study also found that, 
compared with the rates published 10 years ago, there has been a general $30 \%$ increase in the prescription of psychotropic medications in cancer patients, with a greater range of reasons for the prescriptions. The authors suggest that the introduction of shorteracting benzodiazepines has altered physicians' prescription preferences. The National Institutes of Health (NIH) State of the Science Conference on Insomnia [43] concluded that the newer, shorter-acting benzodiazepine receptor agonists (BzRAs) are efficacious in the management of insomnia, and the frequency and severity of adverse effects associated with them are much lower than those seen with the older, longer-acting benzodiazepines. The newer BzRAs and the newest melatonin receptor agonist are listed in Table 1 and described in detail in the specific treatment text that follows. The NIH also concluded that all antidepressants, antihistamines (H1 receptor antagonists), and antipsychotics have potentially significant adverse effects and raised concerns about their risk-benefit ratio and their suitability as treatment of chronic insomnia. Therefore, these drugs are not included in the discussion of treatment options. Clinicians need to evaluate the relative effectiveness and side-effect profiles of pharmacologic agents, and more research needs to be done to evaluate the impact of pharmacologic treatment on sleep disturbances in cancer patients [5••].

\section{Nonpharmacotherapy}

- CBT for insomnia has been shown to be very effective, possibly more effective than pharmacotherapy, in various randomized controlled clinical trials among the general population [44, Class I]. The NIH State of the Science Conference on Insomnia concluded that CBT is as effective as prescription medications for brief treatment of chronic insomnia and that there are indications that the beneficial effects of CBT may last well beyond termination of treatment, in contrast with those produced by medications [43]. Two published studies examined the effect of CBT on insomnia in patients with breast cancer [16••, Class III; 39••, Class I; 45, Class III].

- Quesnel et al. [45, Class III] tested the effects of group CBT on breast cancer patients and found significant improvements in sleep efficiency, total sleep time, mood, and cognitive aspects of quality of life. Savard et al. [16••, Class III; 39••, Class I] conducted a randomized wait-list control study looking at the effects on sleep of group CBT for insomnia in breast cancer survivors, and psychologic and immunologic measures. Objective and subjective sleep measures were recorded, as well as hypnotic medication use, psychologic distress, quality of life, and immune measures that included enumeration of blood cell count and cytokine production. Results showed clear improvements in the subjective sleep data. Significant group-time interactions (two groups $=$ treatment or wait-list control, five times = pretreatment, post-treatment, and 3-, 6-, and 12-month follow-ups) with a priori contrast revealed improvements in all variables except total sleep. The analysis of subjective sleep data pooled from all participants showed significant improvements after treatment in all sleep variables, including sleep-onset latency, wake time, and sleep efficiency. The improvements were maintained for all variables at the 3-, 6-, and 12-month follow-ups, and continued to improve further regarding total sleep time and the insomnia questionnaire filled out by a significant other. More ambivalent results came from the objective PSG data, which showed no significant group-by-time interactions for any variable but showed significant positive changes from pretreatment to post-treatment for all variables except for total sleep time. The changes were maintained at the 6-month follow-up. A significant decrease in hypnotic drug use was also revealed by the participants after being treated and experiencing improvements in psychologic variables (ie, anxiety, depression, global quality of life, and fatigue), and the decrease was maintained at the follow-up assessments [16••, Class III]. As previously discussed, the analysis of 
immunologic measures revealed that at post-treatment participants had higher cytokine production (ie, IL-1- $\beta$, interferon $\gamma$ ) and lower increases of lymphocytes, which then increased at follow-up, as did the leukocyte counts [39••, Class I]. The mediation analysis revealed that some of these changes were partially mediated by improvement in the psychologic- and insomnia-related sleep variables. These results are preliminary but promising and provide some support to the hypothesis that there is a causal relationship between insomnia and immunologic functioning, and that treating insomnia might benefit the immune system.

\section{Combination of pharmacologic and behavioral treatment approaches}

- The combination of pharmacologic and nonpharmacologic therapies was found to produce better short-term improvements than with each therapy alone in non-cancer populations. However, the behavioral therapy alone produced better long-term gains [46]. Despite the empirical evidence in support of the use of CBT for insomnia, this intervention is not often used in clinical practice because few practitioners are trained in the techniques [43] and it requires a time commitment from both patient and practitioner. However, it seems that most of the CBT strategies can be easily and effectively integrated into the cancer care routine [2]. In addition, as reviewed by Theobald [47], treating insomnia in cancer patients with a combination of pharmacologic and nonpharmacologic therapy may have a positive impact not only on the insomnia itself, but also on related symptoms, including pain, fatigue, and psychologic distress.

\section{Future directions}

- In summary, sleep disruption in cancer patients is common and characterized by multifactorial causes. Additional research is needed to determine the baseline levels of quality and quantity in cancer patients. It is possible that the sleep disturbances share common cytokine-based neuroimmunologic mechanisms with other symptoms that are common in cancer patients.

- Although pharmacologic treatments are the most common interventions for sleep disturbances in cancer patients, cognitive-behavioral treatments are very promising and may become gold-standard interventions. Before then, sleep disturbance interventions still need to be tested in cancer patients, and specific modification or developments of new interventions might be necessary to account for the diverse cluster of symptoms expressed in cancer patients. The long-term research goals in regard to sleep disturbances in cancer patients should aim at discovering and elucidating pharmacologic and nonpharmacologic approaches that will improve the quality of life of patients both during and after cancer treatment.

\section{Treatment summary}

\begin{tabular}{|c|c|}
\hline \multicolumn{2}{|l|}{ Specific drugs } \\
\hline Eszopiclone & \\
\hline Standard dosage & 1,2 , or $3 \mathrm{mg}$ administered orally at bedtime. \\
\hline Contraindications & Hypersensitivity to drug or components. \\
\hline Main drug interactions & Alcohol and drugs that have sedating effects. \\
\hline Main side effects & Dizziness and loss of coordination. \\
\hline
\end{tabular}


Special points Nonbenzodiazepine benzodiazepine receptor agonist. Half-life of 6 hours. Must remain inactive for 8 hours. Classified as a schedule IV controlled substance by federal regulation.

Cost/cost effectiveness $\$ 4.40$ per 1-, 2-, or 3-mg tablet.

Ramelteon

$\begin{array}{ll}\text { Standard dosage } & 8 \mathrm{mg} \text { administered orally } 30 \text { minutes before bedtime. } \\ \text { Contraindications } & \text { Hypersensitivity to drug or components. } \\ \text { Main drug interactions } & \begin{array}{l}\text { Alcohol. Fluvoxamine, ketoconazole, and fluconazole increase blood levels of ramelteo } \\ \text { possibly increasing side effects of ramelteon. Rifampin may decrease blood levels of } \\ \text { ramelteon, possibly reducing the effect of ramelteon. }\end{array}\end{array}$

Main side effects Headache, drowsiness, fatigue, dizziness, nausea, worsening of insomnia, diarrhea, and depression.

Special points Melatonin receptor agonist. Not a controlled substance. Should not be taken with or immediately after a high-fat meal because fat increases its absorption from the intestine.

Cost/cost effectiveness $\$ 3.36$ per 8 -mg tablet.

Zaleplon

Standard dosage

Contraindications

Main drug interactions

Main side effects

Special points

Cost/cost effectiveness $\$ 3.76$ per 5 -mg capsule; $\$ 3.86$ per $10-\mathrm{mg}$ capsule.

5,10 , or $20 \mathrm{mg}$ administered orally at bedtime.

Hypersensitivity to drug or components; severe hepatic impairment.

Alcohol. Inhibitors of aldehyde oxidase and (to a lesser extent) of CYP3A4 might be expected to decrease zaleplon's clearance, and inducers of these enzymes might be expected to increase its clearance. Rifampin reduces the amount of zaleplon in the blood by more than $80 \%$. This could lead to a decrease in zaleplon's activity. Cimetidine can greatly increase the amount of zaleplon in the blood; if cimetidine must be used, lower doses of zaleplon are suggested.

Short-term memory impairment, hallucinations, impaired coordination, dizziness, lightheadedness, headache, drowsiness, amnesia, stomach ache, and muscle pain.

Nonbenzodiazepine benzodiazepine receptor agonist. Must remain inactive for 4 hours. Zaleplon is indicated for the short-term treatment of insomnia. Classified as a schedule IV controlled substance by federal regulation.

Zolpidem

Standard dosage

5 or $10 \mathrm{mg}$ administered orally at bedtime indicated for the short-term treatment of insomnia.

Contraindications

Hypersensitivity to drug or components.

Main drug interactions

Main side effects

Alcohol.

Drowsiness, dizziness, and a "drugged" feeling, confusion, insomnia, euphoria, ataxia (balance problems), and visual changes.

Special points Nonbenzodiazepine benzodiazepine receptor agonist. Must remain inactive 7-8 hours Classified as a schedule IV controlled substance by federal regulation.

Cost/cost effectiveness $\$ 5.14$ per 5-mg tablet (generic version will be available in 2007).

Zolpidem controlled-release

Standard dosage

Contraindications

Main drug interactions

Main side effects

Special points

Cost/cost effectiveness
6.5 or $12.5 \mathrm{mg}$ administered orally at bedtime.

Hypersensitivity to drug or components.

Alcohol.

Headache, somnolence (sleepiness), and dizziness.

Nonbenzodiazepine benzodiazepine receptor agonist. Must remain inactive 7-8 hours. Classified as a schedule IV controlled substance by federal regulation.

$\$ 3.86$ per 6.25 - or 12.5 -mg tablet.

CBT

- $\quad$ Five to eight sessions required.

- No known contraindications 
- Brief, goal-oriented psychotherapy, available in individual and group settings.

- Therapy sessions may vary in cost depending on who is conducting the sessions. 


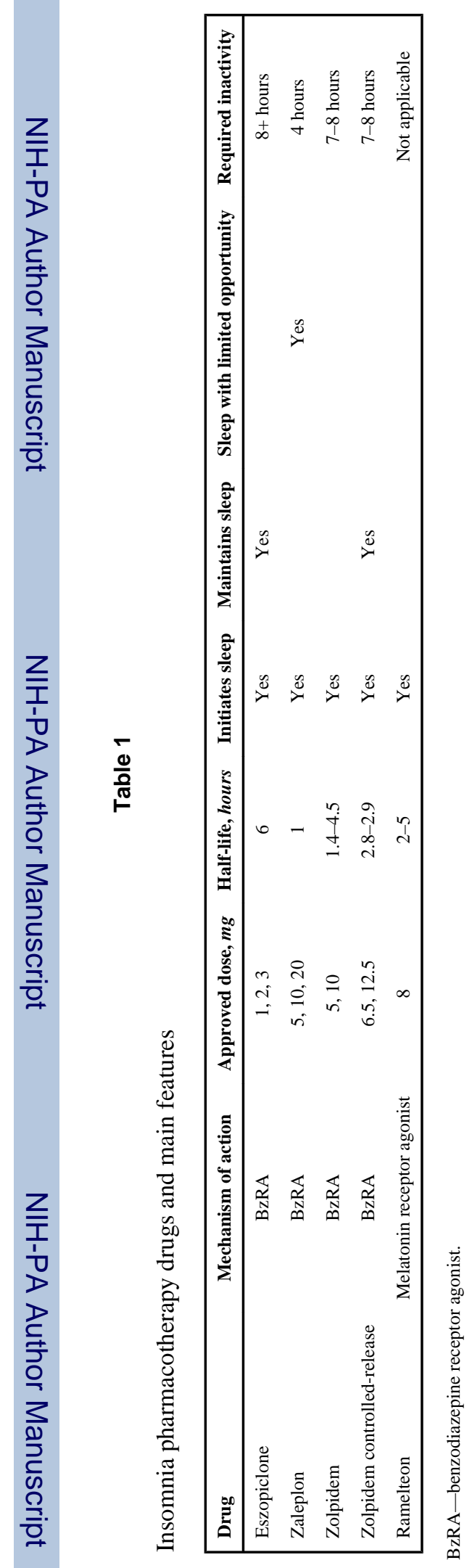

Curr Treat Options Neurol. Author manuscript; available in PMC 2010 October 8. 